\section{LUNG PERFUSION WITH CHEMOTHERAPY IN PATIENTS WITH UNRESECTABLE METASTATIC SARCOMA TO THE LUNG OR DIFFUSE BRONCHIOLOALVEOLAR CARCINOMA}

Eight patients with metastatic sarcoma to the lung $(n=4)$ or diffuse bronchioloalveolar carcinoma of the lung $(n=4)$ underwent isolated lung perfusion with chemotherapy in a pilot study. Ages ranged from 18 to 60 years and half were female. The left lung was perfused in three patients (single lung perfusion) and both lungs in five patients (total lung perfusion). Perfusions ranged from 45 to 60 minutes at ambient or normothermic temperatures. One patient received perfusion at moderate hyperthermia $\left(40^{\circ} \mathrm{C}\right)$. Escalating doses of doxorubicin (1 to $10 \mu \mathrm{g} / \mathrm{ml}$ perfusate) was used in six patients, whereas two received cisplatin (14 and $20 \mu \mathrm{g} / \mathrm{ml}$ perfusate). There were two major complications and no objective responses. The isolated perfusion systems gave excellent separation between systemic and pulmonary circulations with zero to 15\% of the measured peak drug concentration of the pulmonary perfusate detected in the systemic circulation. Drug concentrations in normal lung and tumor generally increased with higher drug dosages and drug was detectable in mediastinal lymph nodes of three out of four patients in whom sampling was done. Isolated lung perfusion with chemotherapy can be done safely in patients with lung malignancies and evidence suggests that higher drug dosages should be well tolerated. (J THORAC Cardiovasc Surg 1995;110:368-73)

Michael R. Johnston, MD, ${ }^{\mathrm{a}}$ Rodney F. Minchen, $\mathrm{PhD},{ }^{\mathrm{b}}$ and Christopher A. Dawson, $\mathrm{PhD},{ }^{\mathrm{c}}$ Toronto, Ontario, Canada, Nedlands, Western Australia, and Milwaukee, Wis.
$R$ egional perfusion and isolated organ perfusion have been investigated as potential methods to deliver high doses of anticancer agents to tumors while limiting the systemic toxicities of the drug. Dose-limiting toxicities develop in many patients undergoing systemic chemotherapy before they have beneficial effects of the therapy. Perfusion may provide more efficacious treatment in those with localized, but unresectable, disease. We have previously described surgical techniques for isolated perfusion of a single lung or both lungs simultaneously. ${ }^{1,2}$ We have also documented both the acute and chronic physiologic consequences of lung perfusion. In separate studies we provided detailed pharmacologic and toxicologic data on the effects of

From the Division of Thoracic Surgery, Mt. Sinai Hospital and the University of Toronto, Toronto, Canada, ${ }^{a}$ the Department of Pharmacology, University of Western Australia, Nedlands, Western Australia, ${ }^{b}$ and the Department of Physiology, Medical College of Wisconsin, Milwaukee, Wis. ${ }^{c}$

Received for publication Aug. 12, 1994.

Accepted for publication Nov. 28, 1994.

Address for reprints: M. R. Johnston, MD, 600 University Ave., Suite 643, Toronto, Ontario, Canada M5G 1 X5.

Copyright $(\mathcal{C} 1995$ by Mosby-Year Book, Inc.

0022-5223/95\$3.00+0 $\mathbf{1 2 / 1 / 6 2 4 6 6}$ doxorubicin (Adriamycin) when administered by lung perfusion ${ }^{3-5}$ and have shown that hyperthermic perfusion is exceptionally well tolerated by the normal lung. 6,7 This study documents our experience in a human trial of lung perfusion. We describe a unique series of eight patients with locally advanced cancers limited to the lung who underwent isolated lung perfusion with either doxorubicin or cisplatin added to the lung perfusate.

\section{Methods}

From 1982 to 1991 eight patients with locally advanced cancers limited to the lung were entered into a pilot trial of lung perfusion with chemotherapy. Patients' ages ranged from 18 to 60 years and there were equal numbers of male and female patients. The initial three patients, all with metastatic soft tissue sarcomas, underwent single lung perfusion with doxorubicin at the Surgery Branch of the National Cancer Institute, Bethesda, Md. The subsequent five perfusions were done at the University of Colorado Health Sciences Center in Denver. Four patients had primary lung cancer and one had metastatic sarcoma. In all five patients total lung perfusion was done during cardiopulmonary bypass, three with doxorubicin and two with cisplatin. Moderate hyperthermia was used in one patient. The study protocol was approved by the Institutional Review Board of both institutions and written informed consent was obtained from each patient. Demographic data on the eight patients are shown in Table I. 
Table I. Demographic data and forced expiratory volume in 1 second in patients undergoing lung perfusion

\begin{tabular}{ccclc}
\hline $\begin{array}{c}\text { Patient } \\
\text { No. }\end{array}$ & $\begin{array}{c}\text { Age } \\
(y r)\end{array}$ & Sex & \multicolumn{1}{c}{ Cell type } & $\begin{array}{c}F E V_{1}(\% \\
\text { predicted })\end{array}$ \\
\hline 1 & 18 & M & Fibrosarcoma & $2.71(60)$ \\
2 & 38 & M & Neurofibrosarcoma & \\
3 & 32 & F & Stromal sarcoma & $2.21(73)$ \\
4 & 53 & F & BAC & $1.07(41)$ \\
5 & 58 & F & BAC & $2.47(95)$ \\
6 & 60 & M & BAC & $2.15(81)$ \\
7 & 58 & M & BAC & $2.46(66)$ \\
8 & 38 & F & Chondrosarcoma & $2.57(91)$
\end{tabular}

$F E V_{I}$, Forced expiratory volume in 1 second; $M$, male; $F$, fermale; $B A C$, bronchioloalveolar carcinoma.

Lung perfusion techniques in the canine model have been previously described in detail. ${ }^{1,2}$ The procedures in human beings are not substantially different, but are generally easier to do because of larger anatomic structures. The following brief description of the precedures gives pertinent differences between canine and human techniques. Specific perfusion parameters for the eight patients are listed in Table II.

Single lung perfusion. All of the canine and human perfusions have been done on the left lung because of easier arterial and venous cannulation. The comparatively longer segment of left pulmonary artery available for securing the arterial cannula and the convenience of using the left atrial appendage for venous cannulation are important considerations in the dog, but probably not as crucial in human beings. Pulmonary artery pressure and perfusate temperature are best monitored by a thermistor-tipped pulmonary artery catheter. It is important to ensure that the perfusion parameters are stable and that the perfusate volume in the reservoir is relatively constant before drug is added to the perfusate. Once drug is added to the perfusate, it is mixed within the extracorporeal circuit for at least 5 minutes before lung perfusion is recommenced. To facilitate this mixing, a shunt line, or "ductus," is included in the tubing circuit and placed as close to the arterial and venous cannulas as feasible. This allows for mixing of the drug in the largest volume of perfusate possible.

Total lung perfusion. This procedure is far simpler in human beings than in the dog. For the systemic perfusion circuit standard cardiopulmonary bypass is used with arterial cannulation of the ascending aorta and a single venous cannula placed through the right atrial appendage. On the pulmonary side the arterial cannula is placed through pursestring sutures directly into the main pulmonary artery. If the pulmonary valve is competent, no clamp or tourniquet is placed proximal to the cannula, even with a pulmonary artery catheter in place. It is important to avoid pulmonary venous hypertension during lung perfusion. Therefore a large (40F) venous cannula is placed through the left atrial appendage. As with the single lung technique, a ductus is included in the tubing circuit to facilitate mixing of the drug. Another length of tubing is
Table II. Lung perfusion parameters

\begin{tabular}{cccccc}
\hline $\begin{array}{c}\text { Patient } \\
\text { No. }\end{array}$ & $\begin{array}{c}\text { Perfusion } \\
\text { method }\end{array}$ & $\begin{array}{c}\text { Flow rate } \\
(\mathrm{ml} / \mathrm{min})\end{array}$ & $\begin{array}{c}P A \\
\text { pressure } \\
(\mathrm{mm} \mathrm{Hg})\end{array}$ & $\begin{array}{c}\text { Perfusate } \\
\text { temperature } \\
\left({ }^{\circ} \mathrm{C}\right)\end{array}$ & $\begin{array}{c}\text { Time } \\
(\text { min })\end{array}$ \\
\hline 1 & Single & 680 & 14 & 27 & 45 \\
2 & Single & 520 & 17 & 27 & 45 \\
3 & Single & 400 & 18 & 35 & 40 \\
4 & Total & 2500 & 12 & 38.7 & 50 \\
5 & Total & 2000 & 25 & 25 & 50 \\
6 & Total & 1200 & 13 & 28.5 & 55 \\
7 & Total & 3000 & 25 & 25 & 50 \\
8 & Total & 2600 & 19 & 40 & 60 \\
\hline
\end{tabular}

$P A$, Pulmonary artery.

placed between the systemic and pulmonary circuits so that perfusate can be easily exchanged between the circuits while the two perfusion systems are being balanced. Once drug is added to the pulmonary perfusate, this line is clamped.

Maintenance of constant volumes in the two perfusion circuits requires interruption of coronary circulation by aortic crossclamping. In every patient an attempt was made to perform lung perfusion with the heart beating and coronary flow intact. Invariably a large volume of perfusate crossed from the systemic to the pulmonary circuit within minutes. With the aorta crossclamped, perfusate volume in the two circuits can be controlled and "balanced" by a few simple maneuvers. Usually the pulmonary circuit tends to gain volume at the expense of the systemic circuit. By a combination of raising flow and perfusion pressure on the pulmonary side and reducing flow and pressure on the systemic side, the two circulations can be balanced as determined by observation of the relative perfusate volume in the two reservoirs. Often, the flow rate on the pulmonary side exceeds the systemic flow rate.

In this study myocardial preservation was achieved with cold blood cardioplegia administered in an antegrade fashion through the aortic root, topical cooling with iced slush in the pericardium, and systemic core cooling to $24^{\circ}$ to $26^{\circ} \mathrm{C}$. Myocardial temperature was carefully monitored with a myocardial probe in the posterior interventricular septum. Keeping the myocardium adequately cooled was sometimes problematic because the pulmonary perfusate returning to the left atrium was between $36^{\circ}$ and $40^{\circ} \mathrm{C}$.

As with the canine perfusions, a left ventricular vent was placed through the apex of the left ventricle with drainage into the pulmonary reservoir. This prevented drug-containing perfusate from collecting in the left ventricle and potentially escaping into the aortic root and coronary arteries through a flaccid aortic valve.

Lung washout. After single lung perfusion residual perfusate was flushed from the lung with $200 \mathrm{ml}$ of systemic blood by releasing the left pulmonary artery tourniquet before clamping the venous cannula. After total lung perfusion the lungs were flushed with 500 to $1000 \mathrm{ml}$ of low-molecular-weight dextran before normal circulation was reestablished.

Drug levels. Samples of systemic blood and pulmonary perfusate were taken every 10 minutes during the perfu- 
Table III. Initial drug dose and drug levels after lung perfusion

\begin{tabular}{ccccccc}
\hline & & \multicolumn{3}{c}{ Drug level } \\
\cline { 3 - 6 } Patient No. & $\begin{array}{c}\text { Drug } \\
\text { (initial dose in } \mu g / m l)\end{array}$ & $\begin{array}{c}\text { Peak } \\
\text { systemic } \\
(\mu \mathrm{g} / \mathrm{ml})\end{array}$ & $\begin{array}{c}\text { Peak } \\
\text { pulmonary } \\
(\mu \mathrm{g} / \mathrm{ml})\end{array}$ & $\begin{array}{c}\text { Lung } \\
(\mu \mathrm{g} / \mathrm{gm})\end{array}$ & $\begin{array}{c}\text { Lymph } \\
\text { Tumor } \\
(\mu \mathrm{g} / \mathrm{gm})\end{array}$ & $\begin{array}{c}\text { node } \\
(\mu \mathrm{g} / \mathrm{gm})\end{array}$ \\
\hline 1 & Doxorubicin (1) & 0.00 & 0.56 & 0.72 & 0.64 & N/A \\
2 & Doxorubicin (1) & 0.00 & 0.56 & 0.79 & 0.25 & N/A \\
3 & Doxorubicin (1.5) & 0.00 & 0.98 & 2.58 & 0.62 & N/A \\
4 & Doxorubicin (3) & 0.23 & 1.46 & 1.58 & 2.19 & N/A \\
5 & Doxorubicin (5) & 0.07 & 2.76 & 2.13 & 1.56 & 0.01 \\
6 & Doxorubicin (10) & 0.17 & 3.08 & 2.81 & 2.81 & 0.08 \\
7 & Cisplatin (14) & 0.44 & 9.34 & 0.69 & 1.42 & 0.84 \\
8 & Cisplatin (20) & 0.80 & 10.15 & 0.68 & 1.09 & 0.00 \\
\hline
\end{tabular}

NIA, Not applicable.

sion and at 20 minutes after perfusion. Lung and tumor tissue samples were taken from representative sites with a stapling device immediately after perfusion. Doxorubicin drug levels were determined by high-pressure liquid chromatography as previously described. ${ }^{3}$ Cisplatin drug levels were determined by digestion of solid tissue in concentrated nitric acid until all organic material was broken down. The subsequent solution was increased to a volume of $10 \mathrm{ml}$ with the addition of deionized water. The perfusate was centrifuged immediately through a centrifree micropartition system to remove proteins above $30,000 \mathrm{kD}$. Both tissue digest and ultrafiltrated perfusate were run on a graphite furnace atomic absorption spectrophotometer and concentrations were obtained by comparison with a standard line.

\section{Results}

All eight patients died of their disease 23 to 151 days after lung perfusion. None had a partial or complete response to therapy; however, judging a radiographic response in patients with diffuse disease was often impossible. There were no intraoperative complications, although on occasion, cannulas were repositioned for optimal perfusion and additional drug was added to counteract the dilutional effect of systemic blood crossing into the pulmonary circuit.

Six of the eight patients were discharged from the hospital within 14 days after perfusion. Two major postoperative complications occurred. In patient 6 a pneumonia that responded promptly to antibiotics and a forceful cough productive of bits of tumor (confirmed by histologic study) developed. A sternal dehiscence developed on about the fourteenth postoperative day, but the rest of the wound remained intact and the sternal nonunion was asymptomatic. No subsequent therapy was required.

Patient 7 was a 58 -year-old man with diffuse bronchioloalveolar carcinoma (BAC), a debilitating cough, and decreased pulmonary function. He previously had unsuccessful systemic doxorubicin therapy and therefore received perfusion with cisplatin. Four days after an uncomplicated total lung perfusion, he required reintubation and mechanical ventilation because of progressive respiratory failure. Respiratory function was eventually stabilized but multiple attempts to wean the patient from the respirator were unsuccessful. Pneumonia and empyema subsequently developed and he died 81 days after perfusion. Postmortem examination revealed massive tumor involvement of the entire lung with no identifiable metastases to lymph nodes or distant sites.

The chemotherapy agent used in the lung perfusion and drug levels obtained from systemic blood, pulmonary perfusate, lung, tumor, and lymph node tissue are presented in Table III. No pharmacologic analysis is possible with this small group of patients and escalating drug dosages. However, certain observations are of interest. The amount of drug entering the systemic circulation was minimal, which indicates the high degree of lung vascular isolation obtained with both techniques. In the three single lung perfusions no drug was detected in the systemic circulation either during the perfusion or after the normal circulation was reestablished. With total lung perfusion some drug crossed over into the systemic circulation, but this averaged only about $7 \%$ (range $2.5 \%$ to $15 \%$ ) of the peak drug level in the pulmonary perfusate.

Pulmonary perfusate drug levels increased appropriately with increasing drug dosages. Drug tissue levels, although showing much more variability, also tended to increase with higher dosages. Mediastinal lymph nodes were sampled immediately after perfusion in the last four patients. Three of the four showed detectable drug levels. In patient 6 there was 
no discrete tumor mass within the lung; however, even grossly normal-appearing lung tissue showed diffuse histologic involvement with bronchioloalveolar carcinoma. Thus lung and tumor drug levels were considered identical. Significant drug levels were found in lung tumor specimens from every other patient.

\section{Discussion}

Once tumor within the lung is found to be unresectable, distant metastatic disease is usually present. Local-regional therapies are then of little value, except to palliate specific symptoms. There are, however, certain notable exceptions. BAC is an uncommon form of lung adenocarcinoma that often grows along alveolar septa without distorting the underlying lung parenchyma. ${ }^{8}$ In its localized form, BAC carries the highest survival rate after surgical resection: up to $80 \%$ at 5 years. ${ }^{9}$ In contrast, the diffuse form of the disease spreads inexorably throughout the lung parenchyma, often without evidence of systemic metastases. In advanced stages of BAC, patients may be incapacitated by cough productive of large amounts of frothy sputum. There is no effective treatment for this form of the disease.

Surgical resection of metastatic sarcoma to the lung has been one of the less-recognized achievements in thoracic surgery. Resection has changed a 2-year survival of zero ${ }^{10}$ to 5-year survival rates of $40 \%$ to $50 \%$ for osteosarcoma ${ }^{11}$ and $20 \%$ to $30 \%$ for soft tissue sarcoma. ${ }^{12,13}$ However, there are a significant number of patients in whom the size or number of metastases in the lung preclude resection. Response to systemic chemotherapy, if any, is invariably transient. Most of these patients are young, otherwise fit, and have no other demonstrable sites of disease.

Both BAC and sarcoma metastatic to the lung offer an opportunity to devise unique local-regional anticancer therapies. Our methods use standard cardiopulmonary bypass technology and equipment to isolate the lung from the systemic circulation. We then perfuse the lung with chemotherapy drugs at concentrations higher than those achieved by intravenous administration.

Perfusion therapy has been used for tumors of brain, liver, and extremities. During the early days of cardiac surgery attempts were made to perfuse lung with use of the emerging technology of cardiopulmonary bypass. ${ }^{14-16}$ Our initial studies rekindled the concept of lung perfusion and described detailed methods for perfusing either one lung or both lungs simultaneously. ${ }^{1,2}$ We also performed dose toxicity studies in rabbit and canine models for doxorubicin, cisplatin, and hyperthermia. ${ }^{3-7}$ Recently, Baciewicz and associates ${ }^{17}$ have expanded on the pharmacokinetics of doxorubicin in lung perfusion and its interaction with heparin. Weksler and associates $^{18,19}$ have devised a model of single-lung perfusion in the rat and performed studies that show growth inhibition of methylcholanthrene-induced sarcoma metastases after doxorubicin perfusion.

Although we previously reported the pharmacokinetic data on our first three perfusion patients, ${ }^{4}$ this is the first study to describe in detail a series of patients treated with chemotherapy by isolated lung perfusion. This study is small and spans a number of years, attesting to the difficulty in convincing institutional review boards, referring physicians, and prospective patients of potential merits of the procedure.

Theoretically, the concentration of drug exposed to tumor by perfusion should be considerably higher than that achievable by intravenous infusion. Although drug delivery by perfusion is difficult to compare with intravenous infusion, by calculating the areas under a concentration versus time curve, estimates of total drug delivery can be made. With intravenous administration there is a high initial peak drug level that rapidly falls off with dilution and a subsequent smaller peak that represents redistribution. Conversely, perfusion permits delivery of high drug levels over a relatively short period, but this in theory results in considerably higher tissue exposure as measured by the area under the concentration versus time curve. Recently Weksler and associates $^{20}$ have shown experimentally that perfusion results in higher lung tissue levels of doxorubicin than intravenous infusion. They isolated and perfused rat lung at various concentrations of doxorubicin, then compared tissue levels with those obtained by intravenous infusion of doxorubicin at doses comparable to those commonly used clinically. With a perfusate drug concentration well tolerated by the rats, lung tissue doxorubicin levels were 20 -fold higher than those with intravenously administered drug.

A major advantage of perfusion therapy is the ability to isolate the lung from the systemic circulation. Thus drugs or drug dosages too toxic to administer by systemic routes might safely be given in the lung. We have shown this to be true for doxorubicin, cisplatin, and hyperthermia. Except for 
some notable pulmonary toxins, such as bleomycin, mitomycin, and cytoxin, many more cytotoxic agents may be applicable for lung perfusion. Even agents too toxic for systemic infusion might be tolerable if limited to the lung.

It was difficult to accrue patients to this study. A combination of its highly experimental nature and invasiveness tended to discourage terminally ill patients, regardless of their physical condition. However, the larger deterrent to both patients and referring oncologists seemed to be the choice of drugs available for perfusion. Many potential patients already had unsuccessful systemic doxorubicin and cisplatin therapy. In others these drugs were not thought to be sufficiently active against the particular tumor to warrant this experimental approach.

In addition, our preclinical laboratory studies were severely limited by the complexities and cost of performing dose-toxicity studies in our canine model. The model recently described by Weksler and Burt ${ }^{18}$ in which lung perfusion is done in a tumor-bearing small animal, may allow for more rapid and cost-effective screening of potential agents. Whether tumor response in this model will be predictive of a similar clinical response remains highly speculative.

Even though the total number of patients perfused in this series is small, some preliminary observations are of note. Most important, there was no major toxicity directly attributable to the lung perfusion at the drug concentrations used. The one patient who died during the same hospital admission had the most symptoms in the group and, in retrospect, was a poor candidate for the procedure. He had previously undergone a left lower lobectomy and had unsuccessful multiple chemotherapy and immunotherapy trials. His pulmonary function was markedly diminished and he required supplemental oxygen with exercise. At autopsy 81 days after perfusion the lungs were almost totally replaced by tumor, suggesting that his death was most likely related to tumor progression. Of interest, he had no detectable nodal or systemic metastases.

The four patients with sarcoma tolerated perfusion exceptionally well and left the hospital between 6 and 10 days after the procedure. Their younger age and better-preserved lung function make this group the obvious choice for future studies. Also, sarcoma may be more responsive to chemotherapy and biologic therapies than BAC.

No patient had a demonstrable tumor response, either in terms of radiographic findings or survival.
Because dose-limiting toxicity was never reached in this pilot study, we cannot speculate as to whether a clinical response might be seen at higher concentrations. However, both canine and small animal studies now suggest that much higher drug dosages can be achieved without untoward toxic effects. $1,17,19,21$

An often-cited criticism of lung perfusion via the pulmonary vasculature relates to tumor blood supply for lung cancers that originate primarily from the bronchial circulation. However, a number of experimental and autopsy studies have shown that a significant portion of both primary and metastatic tumor vasculature is fed by the pulmonary circulation. ${ }^{22,23}$ By measuring significant levels of drug in tumor after isolated perfusion, our study definitively proves in human beings that both primary and metastatic cancers in the lung are at least partially perfused through the pulmonary circulation.

Two methods of lung perfusion were used in this study, single lung perfusion and total lung perfusion. Single lung perfusion has the advantage of less complexity because cardiopulmonary bypass is not needed. Unfortunately, the tumors most suitable for lung perfusion invariably occur bilaterally, thereby requiring two separate procedures to treat the entire lung. This, and the observation that physiologic flow rates (and possibly ideal drug distribution) could not be obtained in single lung perfusions without exceeding normal pulmonary artery pressures, led us to develop the total lung perfusion technique. Both Weksler and associates ${ }^{18}$ and Pogrebniak and colleagues $^{24}$ have recently described laboratory studies that used single lung perfusion to deliver experimental therapies. Perhaps early clinical studies should routinely use this technique to avoid potentially life-threatening bilateral lung damage, but the ultimate clinical utility of lung perfusion will be best served with the total lung perfusion technique.

In conclusion, this highly invasive form of regional cancer treatment combines modern-day surgical techniques with anticancer therapy in an attempt to treat unresponsive local-regional disease. The results of this preliminary trial are encouraging in that the complex procedures seem to be well tolerated and reproducible. Morbidity is certainly well within expectations for a pilot chemotherapy study. Significant levels of drug can be measured in primary lung cancer, metastatic cancer to the lung, and in the regional lymph nodes draining these cancers. 


\section{REFERENCES}

1. Johnston M, Minchin R, Shull J, et al. Isolated lung perfusion with Adriamycin: a preclinical study. Cancer 1983;52:404-9.

2. Johnston M, Christensen C, Minchin R, et al. Isolated total lung perfusion as a means to deliver organspecific chemotherapy: long-term studies in animals. Surgery 1985;98:35-44.

3. Minchin R, Boyd M. The uptake and metabolism of doxorubicin in isolated perfused rat lung. Biochem Pharmacol 1983;32:2829-31.

4. Minchin R, Johnston M, Aiken M, Boyd M. Pharmacokinetics of doxorubicin in isolated lung of dogs and humans perfused in vivo. $\mathbf{J}$ Pharmacol Exp Ther 1984;229:193-8.

5. Bongard RD, Roerig DL, Johnston MR, Linehan JH, Dawson CA. Influence of temperature and plasma protein on doxorubicin uptake by isolated lungs. Drug Metabol Dispos 1993;21:428-34.

6. Rickaby D, Fehring J, Johnston M, Dawson C. Tolerance of the isolated perfused lung to hyperthermia. J Thorac Cardiovasc Surg 1991;101:732-9.

7. Cowen ME, Mulvin DW, Howard RB, Dawson CA, Johnston MR. Lung tolerance to hyperthermia by in vivo perfusion. Eur J Cardiothorac Surg 1992;6:16773.

8. Marchevsky AM. Surgical pathology of lung neoplasms. New York: Marcel Dekker, 1990:112.

9. Daly R, Trastek V, Pairolero P, et al. Bronchoalveolar carcinoma: factors affecting survival. Ann Thorac Surg 1991;51:368-77.

10. Marcove R, Martini N, Rosen G. The treatment of pulmonary metastasis in osteogenic sarcoma. Clin Orthop 1975;111:65-70.

11. Putnam JR, Roth JA, Wesley M, Johnston MR, Rosenberg SA. Survival following aggressive resection of pulmonary metastases from osteogenic sarcoma: analysis of prognostic factors. Ann Thorac Surg 1983; 35:516-23.

12. Putnam JR, Roth JA, Wesley M, Johnston MR, Rosenberg SA. Analysis of prognostic factors in patients undergoing resection of pulmonary metastases from soft tissue sarcomas. J Thorac Cardiovasc SURG 1984;87:260-7.

13. Casson A, Putnam J, Natarajan M, et al. Five-year survival after pulmonary metastasectomy for adult soft tissue sarcoma. Cancer 1992;69:662-8.

14. Creech O Jr, Krementz E, Ryan R, Reemtsma K, Winblad J. Experiences with isolation-perfusion technics in the treatment of cancer. Ann Surg 1959;149: 627-40.

15. Pierpont H, Blades B. Lung perfusion with chemotherapeutic agents. J THORAC CARDIOVASC SURG 1960; 39:159-65.

16. Jacobs J, Flexner J, Scott H. Selective isolated perfusion of the right or left lung. $\mathbf{J}$ THORAC CARDIOvasC SuRG 1961;42:546-52.

17. Baciewicz F, Arredondo M, Chaudhuri B, et al. Pharmacokinetics and toxicity of isolated perfusion of lung with doxorubicin. J Surg Res 1991;50:124-8.

18. Weksler B, Schneide A, Ng B, Burt M. Isolated single lung perfusion in the rat: an experimental model. J Appl Physiol 1993;74:2736-9.

19. Weksler B, Lenert J, Ng B, Burt M. Isolated single lung perfusion with doxorubicin is effective in eradicating soft tissue sarcoma lung metastases in a rat model. J THORAC CARDIOvasC SuRG 1994;107:50-4.

20. Weksler B, Ng B, Lenert J, Burt M. Isolated singlelung perfusion with doxorubicin is pharmacokinetically superior to intravenous injection. Ann Thorac Surg 1993;56:209-14.

21. Minchin R, Johnston M, Schuller J, Aiken M, Boyd M. Pulmonary toxicity of doxorubicin administered by in situ isolated lung perfusion in dogs. Cancer 1988; 61:1320-5.

22. Cudkowicz C, Armstrong J. The blood supply of malignant pulmonary neoplasms. Thorax Lung 1951;8:152-6.

23. Miller B, Rosenbaum A. The vascular supply to metastatic tumors of the lung. Surg Gynecol Obstet 1967;125:1009-16.

24. Pogrebniak $\mathbf{H}$, Witt $\mathbf{C}$, Terrill $\mathbf{R}$, et al. Isolated lung perfusion with tumor necrosis factor: a swine model in preparation of human trials. Ann Thorac Surg 1994; $57: 1477-83$. 